\title{
A Triplet Mechanism for Magnetic Field Modulation of Photochemical Quantum Yields
}

\author{
Ulrich Steiner \\ Institut für Physikalische Chemie der Universität Stuttgart, Pfaffenwaldring 55, D-7000 Stuttgart 80
}

\section{Magnetfeldeffekte / Photochemie / Quantenmechanik / Triplettexciplexe}

A mechanism is described whereby the influence of an external magnetic field on the sublevels of a triplet exciplex, subject to heavy-atom-induced sublevel-selective deactivation, modulates the radical dissociation yield of the exciplex. A sublevel selection rule for the heavy atom effect on $\mathrm{T}_{1}-\mathrm{S}_{0}$ intersystem crossing in the triplex exciplex is derived. The magnetic field influence is treated on the basis of an approximate solution of a stochastic Liouville equation, leading to a simple analytic expression for the magnetic field effect on the radical yield. The result is applied to explain a previously observed magnetic field effect. The mechanism described may induce magnetic field effects on any triplet reaction competing with sublevel-selective deactivation.

\section{Introduction}

Magnetic field effects on chemical and especially photochemical reactions are becoming increasingly important for obtaining detailled information on reaction mechanisms. Reviews of the field are given in Refs. [1-3]. Such effects are closely related to magnetic polarisation phenomena as chemically induced nuclear polarization (CIDNP) and electron spin polarisation (CIDEP) [4-7].

It is a common feature of most of these effects that pairs of paramagnetic particles are involved, such as triplet-triplet pairs, triplet-doublet pairs or doublet-doublet pairs. The mechanistic principle is such that reactions of the paramagnetic pairs lead to states of definite multiplicity (singlet or triplet), i.e. the chemical reaction projects pure spin states out of the manyfold of the pair spin states. For the pair, however, the spin operator $\mathrm{S}^{2}$ does not commute with the spin Hamiltonian, comprising exchange interaction, electronic spin dipolar interaction, hyperfine interaction and Zeeman interaction, so that pure spin states in the pair are mixed by these interactions. Since they include the Zeeman interaction, an external magnetic field will influence transitions between pair states of different multiplicity and can thus modify the product distribution.

Besides the pair mechanism there was a different mechanism shown to operate sometimes in CIDEP, called the triplet mechanism [8-11]. The underlying principle in this case is a selective population of triplet sublevels by intersystem crossing $\left(S_{1} \rightarrow T\right)$ leading to spin polarization in the molecular frame. Spin polarization in the laboratory frame arises if the zero field splitting of the triplet sublevels is not too small with respect to the Zeeman energy.

In a recent preliminary report [12] we gave evidence of a third type of mechanism capable of explaining magnetic field effects on photochemical reactions. It is related in some way to the triplet mechanism of CIDEP and is based on a reaction scheme depicted in Fig. 1. There the sublevels of an excited triplet exciplex decay into free radicals with equal rate constants $\left(k_{\mathrm{fr}}\right)$ but undergo sublevel-selective intersystem crossing $\left(k_{\text {isc }}\right)$ to the singlet ground state. A magnetic field modulates the thermal relaxation among the triplet substates and if the intersystem crossing is fast enough to compete with the thermal relaxation the magnetic field effect on the sublevel repopulation will influence the radical dissociation yield of the exciplex. exciplex triplet
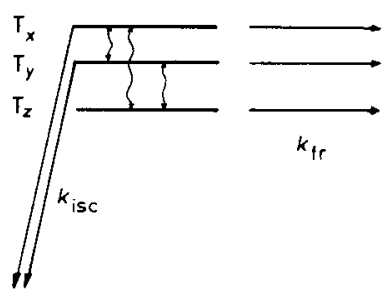

dissociation

into free radicals

to singlet ground state

E4818.1

Fig. 1

Kinetic scheme describing magnetic field effect on triplet exciplex decay. The order of the triplet sublevels $\mathrm{T}_{x}, \mathrm{~T}_{y}, \mathrm{~T}_{z}$ is arbitrary. Wavy arrows between triplet sublevels indicate transitions due to spin-lattice relaxation mechanisms and magnetic field influence

In Ref. [12] we analysed a previously observed heavy-atominduced magnetic field effect [13] making use

i) of the assumption that in the triplet exciplexes investigated the internal heavy atom effect operates for two sublevels only and

ii) of an explicit expression (Eq. (2) in Ref. [12]) for the magnetic field dependence of the effective sublevel relaxation constant.

In this paper we shall give a detailed account of our previous treatment of the mechanism.

Theory

\section{Heavy Atom Induced Sublevel-Selective Intersystem Crossing in Triplet Exciplexes}

As was shown in Ref. [14] triplet exciplexes $\left({ }^{3}\left(A^{*} D^{+}\right)\right)$formed in the reaction of thionine triplet $\left({ }^{3} \dot{A}^{+}\right)$with halogen anilines (D) undergo intersystem crossing to the singlet ground state ${ }^{1}\left(A^{+} D\right)_{0}$ mainly due to the influence of the halogen substituent in the aniline part. From the position dependence of the heavy atom substituent effect one may derive that the charge transfer nature of the exciplex is essential for this type of heavy atom effect. In order to derive selection rules for spinorbit coupling of the individual exciplex triplet sublevels with the singlet ground state we have to regard the intersystem crossing process in the exciplex in detail.

Our treatment of the rate constant $k_{\text {isc }}$ of the radiationless process ${ }^{3}\left(\mathrm{AD}^{*}\right) \sim{ }^{1}\left(\mathrm{~A}^{+} \mathrm{D}\right)_{0}$ will be based on the approximation (Eq. (1)) often applied in the theory of radiationless processes [15]: 
$k_{\text {isc }}=F_{\mathrm{el}} \cdot F_{\mathrm{FC}}$

where $F_{\mathrm{FC}}$ is the Franck-Condon factor and $F_{\mathrm{el}}$ the electronic factor. We assume that triplet exciplex and singlet ground state are directly coupled by the spin-orbit coupling Hamiltonian, so that $F_{\mathrm{el}}$ is proportional to the square of the corresponding matrix element:

$F_{\text {el }} \propto\left|\left\langle^{3}\left(\dot{\mathrm{AD}}^{+}\right)\left|\mathbf{H}_{\text {so }}\right|^{1}\left(\mathrm{~A}^{+} \mathrm{D}\right)_{0}\right\rangle\right|^{2}$.

We assume that the exciplex is of a sandwich type structure providing good overlap between the $\pi$-electron systems of acceptor and donor component (Fig. 2). Since methanol, the solvent used in our ex-

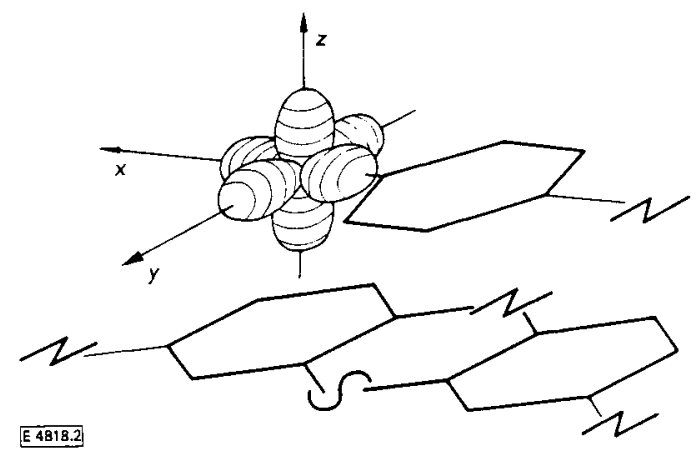

Fig. 2

Assumed geometry of the triplet exciplex between halogen aniline (psubstituted) and thionine. Only p-orbitals of halogen substituent are indicated

periments $[13,14]$ is strongly polar and since the energy of the pure charge transfer state is below the locally excited triplet state [16] we shall approximate the triplet exciplex as a pure charge transfer state. In order to calculate the matrix element in Eq. (2) we represent the exciplex state by a two-electron wave-function, corresponding to the configuration ${ }^{3}(a d)$, where $a$ is the lowest unoccupied molecular $\pi$-orbital of the acceptor and $d$ is the highest occupied molecular $\pi$ orbital of the donor. The exciplex ground state configuration will be correspondingly represented by ${ }^{1}(d d)$. Thus Eq. (2) becomes:

$F_{\mathrm{el}} \propto\left|\left\langle^{3}(a d)\left|\mathbf{H}_{\mathrm{so}}\right|^{1}(d d)\right\rangle\right|^{2}$

Eq. (3) may be interpreted as describing a transition of the electron from the acceptor orbital $a$ to the donor orbital $d$, accompanied by a spin-flip induced by spin-orbit coupling. Since it is almost exclusively the heavy atom influence which is observed in the intersystem crossing rate constant of the systems investigated $[13,14]$ we approximate the spin-orbit coupling operator by the contribution of the heavy atom center only.

$\mathbf{H}_{\mathrm{so}}=\sum_{i=\mathbf{1}, 2} A\left(r_{i}\right)\left(\mathbf{l}_{x i} \mathbf{s}_{x i}+\mathbf{l}_{y i} \mathbf{s}_{y i}+\mathbf{l}_{z i} \mathbf{s}_{z i}\right)$.

In Eq. (4) $A\left(r_{i}\right)$ is a rapidly decaying function of the distance $r_{i}$ of the electron $i$ from the heavy atom nucleus, describing the screeening of the heavy atom nuclear charge by the heavy atom electrons [17]. The $\mathbf{I}_{\nu}$ and $\mathbf{s}_{y}(v=x, y, z)$ are the components of the orbital and spin angular momentum operators of the two electrons considered explicitely in the wave functions. Since the operator is centered at the heavy atom it will be convenient to use the local symmetry at the heavy atom substituent site for defining the directions $x, y$, and $z$. Therefore we identify these directions with the axes of the halogen p-orbitals (Fig. 2).

Integrating over the spin coordinates of both electrons and over the spatial coordinates of one of the electrons we obtain:

$F_{\mathrm{el}}\left(\mathrm{T}_{v}\right) \propto\left|\left\langle a\left|A(r) l_{v}\right| d\right\rangle\right|^{2}$

where we have neglected overlap between molecular orbitals $a$ and $d$. As can be seen each component $\mathbf{l}_{v}$ of the orbital angular momentum operator is responsible for the coupling of one triplet substrate of the corresponding symmetry. In the substate $T_{v}$ the spin is quantitized perpendicular to the axis $v$. It should be noted that these substates are not necessarily the ones diagonalizing the zero field Hamiltonian, which is governed by dipolar spin-spin interaction depending on the overall symmetry of the exciplex.

Integrating the matrix element in Eq. (5) one can make use of the rapid decay of $A(r)$ so that only those parts of the orbitals $a$ and $d$, extending to the heavy atom will contribute to the matrix element [18]. Hence we may replace the orbital $d$ by the function $c_{d, p_{z} \text {, ha }} \cdot p_{z \text {, ha }}$, where $c_{d, p_{z} \text {, ha }}$ is the corresponding MO-coefficient of the heavy atom in the orbital $d$ and $p_{z, \text { ha }}$ the heavy atom valence $p_{z}$-orbital. The acceptor orbital $a$ may be replaced by its projection $a_{\mathrm{ha}}$ onto the heavy atom AO's

$F_{\mathrm{el}}\left(\mathrm{T}_{v}\right) \propto\left|\left\langle a_{\mathrm{ha}}\left|A(r) \mathbf{I}_{\nu}\right| p_{z, \mathrm{ha}}\right\rangle\right|^{2} c_{d, p_{z}, \mathrm{ha}}^{2}$.

In proceeding from Eqs. (5) to (6) we neglected all non-one-center contributions to the matrix element. Applying the angular momentum operator $\mathbf{l}_{\nu}$ yields for the individual sublevels the following expressions for $F_{\mathrm{el}}$ :

$$
\begin{aligned}
F_{\mathrm{el}}\left(\mathrm{T}_{x}\right) & \propto\left|\left\langle a_{y, \mathrm{ha}}|A(r)| p_{y, \mathrm{ha}}\right\rangle\right|^{2} c_{d, p_{z}, \mathrm{ha}}^{2} \\
F_{\mathrm{el}}\left(\mathrm{T}_{y}\right) & \propto\left|\left\langle a_{x, \mathrm{ha}}|A(r)| p_{x, \mathrm{ha}}\right\rangle\right|^{2} c_{d, p_{z}, \mathrm{ha}}^{2} \\
F_{\mathrm{el}}\left(\mathrm{T}_{z}\right) & =0 .
\end{aligned}
$$

Since $\mathrm{l}_{z} p_{z}=0$ the heavy atom does not contribute to the intersystem crossing rate constant of $\mathrm{T}_{z}$. The heavy atom effect on $F_{\mathrm{el}}$ for $\mathrm{T}_{x}$ and $\mathrm{T}_{y}$ depends on the functions $a_{y, \text { ha }}$ and $a_{x, \text { ha }}$ which are the contributions to $a_{\text {ha }}$ with the same angular dependence as $p_{y}$ and $p_{x}$ respectively. The most important contribution to $a_{x, \text { ha }}$ and $a_{y, \text { ha }}$ will be from the atomic valence orbitals $p_{x \text {, ha }}$ and $p_{y, \text { ha }}$. These contributions depend on the overlap between the halogen atomic orbitals and the acceptor MO $a$ and thus on the geometry of the exciplex. Though presently we have no definite knowledge of the exciplex geometry we consider it reasonable that the overlap of $p_{x, \text { ha }}$ and $p_{y, \text { ha }}$ with the acceptor MO $a$ will be of the same order of magnitude.

Therefore we shall assume:

$F_{\mathrm{el}}\left(\mathrm{T}_{x}\right)=F_{\mathrm{el}}\left(\mathrm{T}_{y}\right)$

Setting $a_{x, \text { ha }}=$ const. $\cdot p_{x, \text { ha }}$ and $a_{y, \text { ha }}=$ const. $\cdot p_{y, \text { ha }}$ and assuming that the Franck-Condon factor $\left(F_{\mathrm{FC}}\right.$ in Eq. (9)) does not vary in the series of compounds considered, we obrain:

$k_{\text {isc }}\left(\mathrm{T}_{x, y}\right) \propto \zeta_{\mathrm{ha}}^{2} c_{d, p_{z}, \mathrm{ha}}^{2}$

with $\zeta_{\text {ha }}$ the atomic spin-orbit coupling constant of the halogen atom. Eq. (9) represents the position dependent heavy atom effect as observed qualitatively in Ref. [14].

\section{The Dynamical Problem \\ Outline}

The appropriate theoretical basis to describe the dynamical problem represented by the scheme in Fig. 1 is provided by the following stochastic Liouville equation for the density matrix of the exciplex triplet:

$$
\begin{aligned}
\dot{\rho}(\Omega)= & \mathrm{i}\left[\rho(\Omega), \mathbf{H}_{\mathrm{Z}}+\mathbf{H}_{\mathrm{D}}(\Omega)\right]_{-}+D_{\mathrm{r}} \nabla^{2} \rho(\Omega)-\left(k_{\mathrm{fr}}+k_{\mathrm{isc}}\right) \rho(\Omega) \\
& +\frac{1}{2} k_{\mathrm{isc}}\left[\mathbf{P}_{\mathrm{T}_{\mathbf{z}^{\prime}}}(\Omega), \rho(\Omega)\right]_{+}
\end{aligned}
$$

The various terms on the right hand side in Eq. (10) describe the time dependence of the density matrix $\rho(\Omega)$ due to different processes. Since the spin motion and the intersystem crossing probability depend on the relative orientation of spin, magnetic field and molecular axes it is necessary to consider the density matrix as a function of molecular orientation, where $\Omega$ is a short-hand notation for the Eulerian angles $(\alpha, \beta, \gamma)$ relating the molecule-fixed coordinate system $\left(x^{\prime}, y^{\prime}, z^{\prime}\right)$ to a laboratory fixed system $(x, y, z)$. The first term, $[\mathrm{A}, \mathrm{B}]_{-}$denoting the commutator $(\mathrm{AB}-\mathrm{BA})$, describes the spin motion due to the spinHamiltonian involving Zeeman interaction $\left(\mathbf{H}_{\mathbf{Z}}\right)$ and electronic dipolar spin-spin interaction $\left(\mathbf{H}_{\mathrm{D}}(\Omega)\right)$, the latter depending on the molecular orientation if laboratory fixed basis states are chosen. The second term on the right hand side describes the rotational diffusive motion of the exciplex, where $D_{\mathrm{r}}$ is the rotational diffusion coefficient and $\nabla^{2}$ is the generalized Laplace operator $[19,20]$ acting on functions of the angular variables $\Omega$. 
Whereas the first two terms on the right hand side of $\mathrm{Eq}$. (10) leave the trace of the density matrix, averaged over $\Omega$, unchanged, thus in effect describing the relaxation among the triplet substates, the third and fourth term describe the decay of the triplet exciplex due to intersystem crossing $\left(k_{\mathrm{isc}}\right)$ and dissociation into radicals $\left(k_{\mathrm{fr}}\right) . P_{\mathrm{T}_{z^{\prime}}}(\Omega)$ is a projection operator projecting onto the $T_{z^{\prime}}$ state (quantized perpendicularly to $z^{\prime}$, the $z$-axis in the molecular frame). The anticommutator, $[A, B]_{+}=(A B+B A)$, is used to preserve the Hermitian property of the density matrix. An exact solution of the stochastic Liouville Eq. (10) is presently being worked out, using methods described by Freed et a]. [21]. In this paper we shall describe the derivation of an approximate solution applied previously [12] to analyse the experimental data of Ref. [13] and which can be given an illustrative physical interpretation (see below).

Our approximation starts from the assumption that spin motion and molecular diffusive rotation may be treated independent of the decay processes and, since they are responsible for the relaxation among the triplet sublevels, their influence may be described by a set of rate constants for transitions between the triplet substates. After these rate constants are determined, they will be combined with the decay constants of the exciplex to an overall kinetic scheme allowing calculation of the radical yield from dissociation of the exciplex.

\section{Triplet Sublevel Relaxation}

For convenience we consider a situation where at $t=0$ we have population of the $T_{z^{\prime}}$-state only and describe the motion of the density matrix in the basis $\left(\mathrm{T}_{+}, \mathrm{T}_{0}, \mathrm{~T}_{-}\right)$of states quantized with respect to the magnetic field direction. The influence of the diffusional rotation of the exciplex described by the operator $D_{r} \nabla^{2}$ in $\mathrm{Eq} .(10)$ is to rotate the molecular coordinate system and hence to change the spin state when described within this molecular basis. On the other hand spin motion with respect to the laboratory and molecular frame is induced by the spin-Hamiltonian comprising Zeeman and electron spin-spin dipolar interaction. The strength of the latter may be estimated by the $D$-parameter, characterizing the zero-field-splitting of the exciplex triplet sublevels. For charge transfer states as in our exciplex case the $D$-parameter is expected to be in the order of 200 Gauss $[22-24]$ corresponding to a Larmor frequency of $3.5 \cdot 10^{9} \mathrm{rad} \mathrm{s}^{-1}$. On the other hand the influence of the diffusive reorientations may be estimated by Debye's formula for the molecular orientational correlation time $\tau_{\mathrm{r}}$ [25]:

$$
\frac{1}{\tau_{\mathrm{r}}}=6 D_{\mathrm{r}}=\frac{3 k T}{4 \pi \eta a^{3}}
$$

where $k, T, \eta, a$ are Boltzmann's constant, absolute temperature, solvent viscosity and the molecular hydrodynamic radius of the exciplex. Assuming a hydrodynamic radius of the exciplex of $4 \dot{A}$ we obtain for methanol at room temperature $1 / \tau_{\mathrm{r}}=25 \cdot 10^{9} \mathrm{~s}-1$. Thus we see that the influence of the diffusive reorientation is much stronger than the influence of the dipolar spin-spin interaction and to a first approximation we may neglect $\mathbf{H}_{\mathrm{D}}$ with respect to $D_{\mathrm{r}} \nabla^{2}$. Thus we are left with the problem to solve the equation

$\dot{\rho}(\Omega)=\mathrm{i}\left[\rho(\Omega), \mathbf{H}_{\mathrm{Z}}\right]_{-}+D_{\mathrm{r}} \nabla^{2} \rho(\Omega)$.

The Zeeman Hamiltonian is given by

$\mathbf{H}_{\mathrm{Z}}=\frac{g \mu_{0}}{h} B \mathrm{~S}_{z}$.

We have chosen the $z$-axis parallel to the magnetic field. $B$ is the magnetic flux density, $S_{z}$ is the $z$-component of the electron spin operator and the meaning of the other symbols is as usual.

We are not really interested in the complete density matrix but only in the probability to find the exciplex in the $T_{z^{\prime}}$ state where $z^{\prime}$ is the molecular $z$-axis. The probability $p\left(\mathrm{~T}_{z^{\prime}}\right)$ is given by

$p\left(\mathrm{~T}_{z^{\prime}}\right)=\int_{\Omega} \operatorname{tr}\left\{\mathbf{P}_{\mathrm{T}_{z^{\prime}}}(\Omega) \rho(\Omega) ; \mathrm{d} \Omega\right.$

where tr ${ }_{\text {d }}$ denotes the trace operation and $\mathbf{P}_{\mathrm{T}^{\prime}}$ is a projection operator projecting onto the $T_{z^{\prime}}$ state. The trace has to be integrated over all orientations $\Omega$ of the exciplex. The orientational dependence of
$\mathbf{P}_{\mathrm{T}_{z^{\prime}}}(\Omega)$ is most conveniently expressed using irreducible tensor operators [26] given in Eqs. $(15 \mathrm{a}-\mathrm{d})$ in terms of the spin operators $\mathbf{S}^{2}$, $\mathbf{S}_{z}, \mathbf{S}_{+}, \mathbf{S}_{-}$.

$\mathbf{S}_{0}^{(0)}=\frac{1}{2} \mathbf{S}^{2} ; \quad \mathbf{S}_{0}^{(2)}=\sqrt{6}\left(\mathbf{S}_{z}^{2}-\frac{1}{3} \mathbf{S}^{2}\right)$

$\mathbf{S}_{ \pm 1}^{(2)}=\mp\left(\mathbf{S}_{z} \mathbf{S}_{ \pm}+\mathbf{S}_{ \pm} \mathbf{S}_{z}\right) ; \quad \mathbf{S}_{ \pm 2}^{(2)}=\mathbf{S}_{ \pm} \mathbf{S}_{ \pm}$.

It can be easily verified that, for $\Omega=(0,0,0), \mathbf{P}_{\mathrm{T}_{z^{\prime}}}(\Omega)$ is given by

$\mathbf{P}_{\mathrm{T}_{z^{\prime}}}(0,0,0)=\frac{1}{3} \mathbf{S}_{0}^{(0)}-\frac{1}{\sqrt{6}} \mathbf{S}_{0}^{(2)}$

which on rotation of the exciplex is transformed as

$\mathbf{P}_{\mathrm{T}_{z^{\prime}}}(\Omega)=\frac{1}{3} \mathbf{S}_{0}^{(9)}-\frac{1}{\sqrt{6}} \sum_{k=-2}^{2} \mathbf{S}_{k}^{(2)} R_{k 0}^{(2)}(\Omega)$.

The $R_{m m^{\prime}}^{(j)}(\Omega)$ are the elements of the Wigner rotation matrices [27]. It should be noted that these matrix elements represent a complete orthogonal basis of the functions over the space $\Omega$ [26]. The orthogonality relation is given by:

$\int_{\Omega} R_{k l}^{(j)} R_{k^{\prime} l^{\prime}}^{\left(j^{\prime}\right)^{*}} \mathrm{~d} \Omega=\delta_{k k^{\prime}}, \delta_{l \prime^{\prime}} \delta_{j j^{\prime}} \frac{8 \pi^{2}}{2 j+1}$.

Starting from a situation where the orientational distribution of the molecules is completely random but only the $\mathrm{T}_{z^{\prime}}$-state is populated the density matrix at time zero $(\rho(\Omega, 0))$ equals the projection operator $\mathbf{P}_{T_{z^{\prime}}}(\Omega)$ times a normalization factor $1 / 8 \pi^{2}$. Using the symmetry property of the $R^{(j)}$ matrices [27] it may be written in the following form:

$\rho(\Omega, 0)=\frac{1}{8 \pi^{2}}\left(\frac{1}{3} \mathbf{S}_{0}^{(0)}-\frac{1}{\sqrt{6}} \sum_{k=-2}^{2}(-1)^{k} \mathbf{S}_{-k}^{(2)} R_{k 0}^{(2)^{*}}\right)$.

One of the main advantages of using the generalized spherical harmonics $R_{m m^{\prime}}^{(j)}(\Omega)$ is that they are eigenfunctions of the operator $D \nabla^{2}$ with the eigenvalue $-j(j+1)[19,26]$ :

$D_{\mathrm{r}} \nabla^{2} R_{m m^{\prime}}^{(j)}(\Omega)=-D_{\mathrm{r}} j(j+1) R_{m m^{\prime}}^{(j)}(\Omega)$.

Thus it is readily seen that

$$
\begin{aligned}
\rho(\Omega, t)= & \frac{1}{8 \pi^{2}}\left(\frac{1}{3} \mathrm{~S}_{0}^{(0)}\right. \\
& \left.-\frac{1}{\sqrt{6}} \mathrm{e}^{-6 D_{\mathrm{r} t}} \sum_{k=-2}^{2}(-1)^{k} \mathrm{e}^{-\mathrm{i} \mathbf{H}_{\mathrm{Z}^{t}}} \mathrm{~S}_{-k}^{(2)} \mathrm{e}^{\mathrm{i} \mathbf{H Z}^{t} R_{k o}^{(2)^{*}}}\right)
\end{aligned}
$$

is the solution of Eq. (12) with the initial condition given by Eq. (19). Inserting Eqs. (17) and (21) into Eq. (14) and making use of the orthogonality relation (18) one obtains for the probability $p\left(\mathrm{~T}_{z^{\prime}}\right)$ at time $t$ :

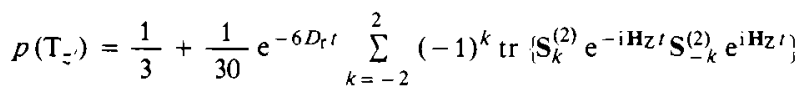

which is easily evaluated in a basis diagonalizing the Zeeman Hamiltonian. One obtains:

$p\left(\mathrm{~T}_{z^{\prime}}\right)=\frac{1}{3}+\frac{1}{30} \mathrm{e}^{-6 D_{\mathrm{r}} t}\left(\sum_{i j k} \mathrm{e}^{-1\left(\omega_{j}-\omega_{i}\right) t}\left\langle i\left|\mathbf{S}_{k}^{(2)}\right| j\right\rangle^{2}\right)$

with

$\omega_{1}=\omega, \quad \omega_{0}=0, \quad \omega_{-1}=-\omega$

$\omega=\frac{\mu_{0} g B}{\hbar}$.

The final result is:

$p\left(\mathrm{~T}_{z}\right)=\frac{1}{3}+\frac{2}{15} \mathrm{e}^{-6 D_{r} t}(1+2 \cos (\omega t)+2 \cos (2 \omega t))$. 


\section{A Vector Model for Triplet Sublevel Relaxation}

The result given in Eq. (26) may be rationalized as follows. Since in a spin state $\mathrm{T}_{z}$ the spin is oriented perpendicularly to the $z$-axis it may be represented graphically as shown in Fig. 3, where the arrows indicate possible spin orientations in the $x y$-plane and the $z$-axis is perpendicular to the spin orientation.

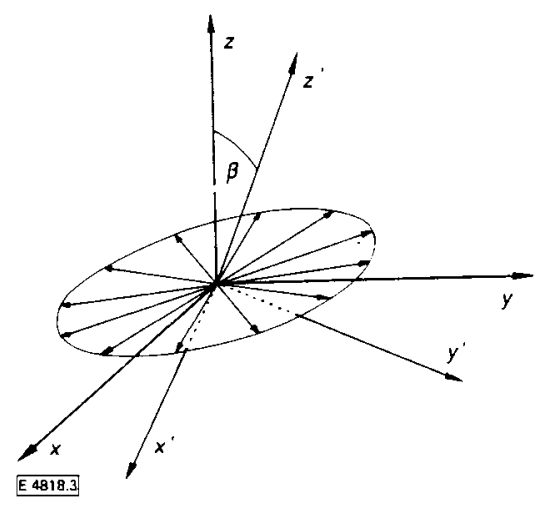

Fig. 3

Representation of possible spin orientation in substate $T_{z}$. The spin vector (arrows) is restricted to lie in the $x y$-plane. In the approximation applied the spin can be assumed to remain unchanged in the laboratory-fixed coordinates $(x, y, z)$ when the orientation of the molecule-fixed axes changes from $(x, y, z)$ to $\left(x^{\prime}, y^{\prime}, z^{\prime}\right)$ due to rotational diffusion

Our assumption that the dipolar spin-spin interaction may be neglected as compared to the influence of rotational diffusion essentially means that the spin orientation in the laboratory frame does not change with molecular reorientation. In Fig. 3 the orientation of the molecular axis at time zero is indicated by $x, y$ and $z$, whereas the primed symbols indicate the molecular axes at some later time. Starting with an initial situation where $T_{z^{\prime}}=T_{z}$ the probability to find the system in the state $T_{z^{\prime}}$ after the $z^{\prime}$-axis has been tilted by an angle $\beta$ is given by $\cos ^{2} \beta$. Due to the diffusional rotation of the molecule the average of $\cos ^{2} \beta$ decays as $\exp \left(-6 D_{\Gamma} t\right)$ to a constant value of $1 / 3$, i. e.

$p\left(\mathrm{~T}_{z^{\prime}}\right)=p\left(z / / z^{\prime}\right)=\frac{1}{3}+\frac{2}{3} \mathrm{e}^{-6 D_{\mathrm{r}} t}$

where the symbol $p\left(z / / z^{\prime}\right)$ denotes the length ${ }^{2}$ of the projection of the unit vector along $z$ onto $z^{\prime}$. Since $p\left(\mathrm{~T}_{z^{\prime}}\right), p\left(\mathrm{~T}_{x^{\prime}}\right)$ and $p\left(\mathrm{~T}_{y^{\prime}}\right)$ have to sum to unity and since for isotropic rotational diffusion $p\left(\mathrm{~T}_{x^{\prime}}\right)=p\left(\mathrm{~T}_{y^{\prime}}\right)$ we obtain

$\left.\begin{array}{l}p\left(\mathrm{~T}_{x^{\prime}}\right)=p\left(\mathbf{2} / x^{\prime}\right) \\ p\left(\mathrm{~T}_{y^{\prime}}\right)=p\left(\mathbf{z} / y^{\prime}\right)\end{array}\right\}=\frac{1}{3}-\frac{1}{3} \mathrm{e}^{-6 D_{\mathrm{r}} t}$.

Next we consider the influence of a magnetic field on the $T_{z}$-state. Since the exciplex molecules are oriented randomly the $z$-axis of the molecule (equal to $z^{\prime}$ at $t=0$ ) will be at some angle $\theta$ to the magnetic field direction (Fig. 4). In this case $\mathrm{T}_{z}$ is not an eigenstate of the Zeeman Hamiltonian and is represented by a superposition of the eigenstates $T_{+}, T_{0}, T_{-}$having different energies and hence different phase velocities. Hence $T_{z}$ becomes time dependent as described by:

$\left|\mathrm{T}_{z}(t)\right\rangle_{\theta}=\frac{1}{\sqrt{2}} \sin \theta \mathrm{e}^{-\mathrm{i} \omega t}\left|\mathrm{~T}_{+}\right\rangle+\cos \theta\left|\mathrm{T}_{0}\right\rangle-\frac{1}{\sqrt{2}} \sin \theta \mathrm{e}^{\mathrm{i} \omega t}\left|\mathrm{~T}_{-}\right\rangle$.

The probability that after some time $t$ the system is still in the initial state $\mathrm{T}_{z}(l=0)$ is given by

$p_{\theta}\left(\mathrm{T}_{z}(t)=\mathrm{T}_{z}(0)\right)=\left(\cos ^{2} \theta+\sin ^{2} \theta \cos \omega t\right)^{2}$.

The spin motion described by Eq. (29) may by visualized as a precession of the axis $z^{\prime \prime}$, defined to be perpendicular to the spin orien-

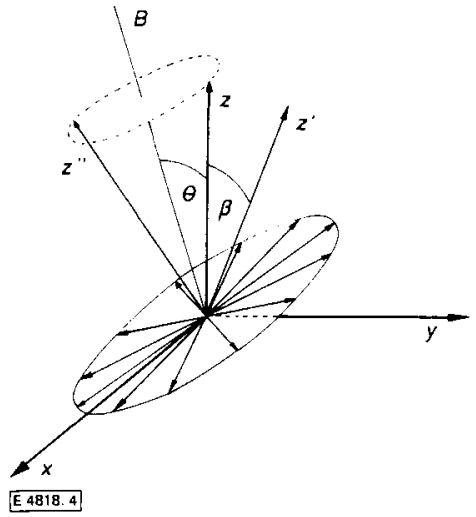

Fig. 4

Representation of the effects of magnetic field and rotational diffusion on spin state $\mathrm{T}_{z}$. The axes $x, y, z$ are laboratory-fixed. At $t=0$, the molecule-fixed axis $z^{\prime}$ and the axis $z^{\prime \prime}$, perpendicular to spin orientation, are parallel to $z$. The effect of the magnetic field (at an angle $\theta$ to $z$ ) on the plane of spin orientation is described by a precession of $z^{n}$ around the field direction. The motion of the molecule-fixed axis $z^{\prime}$ which is due to rotational diffusion is uncorrelated with the motion of $z^{\prime \prime}$

tation, around the magnetic field direction (see Fig. 4). Averaging over all possible angles $\theta$ of the magnetic field we obtain:

$p\left(\mathrm{~T}_{z}\right)=p\left(z^{\prime \prime} / / z\right)=\frac{1}{15}(7+4 \cos (\omega t)+4 \cos (2 \omega t))$.

Since $p\left(z^{\prime \prime} / / z\right), p\left(z^{\prime \prime} / / x\right)$ and $p\left(z^{\prime \prime} / / y\right)$ must sum to one, and for symmetry reasons $p\left(z^{\prime \prime} / / x\right)$ averaged over all angles $\theta$ must equal $p\left(z^{n} / / y\right)$ we obtain:

$\left.\begin{array}{l}p\left(\mathrm{~T}_{x}\right)=p\left(z^{\prime \prime} / / x\right) \\ p\left(\mathrm{~T}_{y}\right)=p\left(z^{\prime \prime / / y)}\right.\end{array}\right\}=\frac{1}{15}(4-2 \cos (\omega t)-2 \cos (2 \omega t))$.

In our approximation spin motion (represented by axis $z^{\prime \prime}$ ) and molecular reorientational motion (represented by $z^{\prime}$ ) are completely uncorrelated processes. Hence their combined effect may be easily calculated. The probability to find the system in the (molecular fixed) $\mathrm{T}_{z^{\prime}}$ state at time $t$, when it was in $\mathrm{T}_{z^{\prime}}$ at time $t=0$, equals the projection of the unit vector along $z^{\prime \prime}$ onto the axis $z^{\prime}$. This quantity may be decomposed into three components as given by Eq. (33):

$$
\begin{aligned}
p\left(\mathrm{~T}_{z^{\prime}}\right)= & p\left(z^{\prime} / / z^{\prime \prime}\right)=p\left(z^{\prime} / / x\right) p\left(z^{\prime \prime} / / x\right)+p\left(z^{\prime} / / y\right) p\left(z^{\prime \prime} / / y\right) \\
& +p\left(z^{\prime} / / z\right) p\left(z^{\prime \prime} / / z\right) .
\end{aligned}
$$

Inserting Eqs. (28), (29) and (31), (32) into Eq. (33) we obtain the result of Eq. (26).

\section{Rate Constant Approximation of Magnetic Field Effect}

The time evolution of the triplet sublevel population in the absence of decay processes as given by Eq. (26) is depicted in the diagram Fig. 5. As can be seen the exponential decay to the equilibrium population is modulated by the magnetic field such that the mean overpopulation of the substate $T_{z^{\prime}}$ is less in a magnetic field. Of course such an oscillating relaxation towards equilibrium cannot be exactly described by a kinetic scheme with time-independent rate constants. In order, however, to proceed in a simple way we replace the oscillating relaxation behaviour in the presence of a field by an effective monoexponential process, which is chosen such that the mean overpopulation during the approach towards equilibrium is the same as in the case described by Eq. (26). Hence our effective relaxation rate constant $r$ in the presence of a magnetic field will be calculated by use of the equation:

$\int_{0}^{\infty} p\left(\mathrm{~T}_{z^{\prime}}\right) \mathrm{d} t=\int_{0}^{\infty}\left(\frac{1}{3}+\frac{2}{3} \mathrm{e}^{-r t}\right) \mathrm{d} t$ 


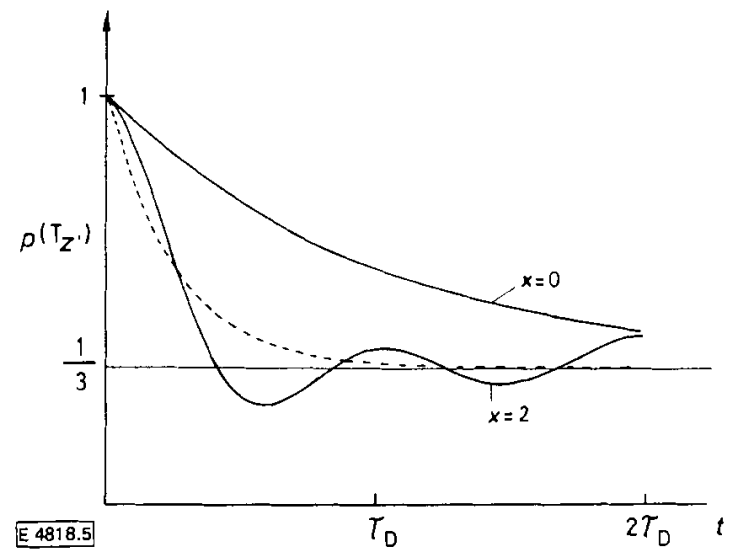

Fig. 5

Relaxation behaviour of population $p\left(\mathrm{~T}_{z^{\prime}}\right)$ of $\mathrm{T}_{z^{\prime}}$ (corresponding to $\mathrm{T}_{z^{-}}$ substate in molecular coordinate system, cf. Fig.2) for different values of parameter $x$ (Eq. (36)) corresponding to different magnetic flux densities. The dashed line represents the effective first order relaxation behaviour obtained using Eq. (35)

The result is:

$r=6 D_{\mathrm{r}} \frac{1+5 x^{2}+4 x^{4}}{1+3 x^{2}+\frac{4}{5} x^{4}}$

with the parameter $x$ given by:

$x=\frac{\omega}{6 D_{\mathrm{r}}}$

The radical yield due to the dissociation of the triplet exciplex can now be calculated from the kinetic scheme given in Fig. 1, where the transitions between the triplet substates are assumed to be first order rate processes with rate constants $1 / 3 r$, so that equilibrium between the substates is established with an effective rate constant $r$. Assuming that in the electron transfer reaction between dye triplet and donor the exciplex triplet sublevels are populated equally we obtain for the radical yield:

$\Phi_{\mathrm{fr}}=\frac{k_{\mathrm{fr}}\left(k_{\mathrm{fr}}+\frac{1}{3} k_{\mathrm{isc}}+r\right)}{\left(k_{\mathrm{fr}}+k_{\mathrm{isc}}\right)\left(k_{\mathrm{fr}}+\frac{2}{3} r\right)+\frac{1}{3} r k_{\mathrm{fr}}}$.

Eqs. (35) - (37) provided the basis for our previously reported analysis [12] of the heavy atom induced magnetic field effect on the radical yield of electron transfer reactions between thionine triplet and halogen substituted anilines [13]. The experimental results are well explained by these relations if the dissociation rate constant $k_{\mathrm{fr}}$ for the exciplex is assumed to be about $1 \cdot 10^{9} \mathrm{~s}^{-1}$ and $k_{\text {isc }}$ is determined by Eq. (37) from $\Phi_{\mathrm{fr}}$ measured at zero field.

The relative magnetic field effect $R$ as shown in Figs. 6 and 7 is defined as

$R=\frac{\Phi_{\mathrm{fr}}(B)-\Phi_{\mathrm{fr}}(0)}{\Phi_{\mathrm{fr}}(0)}$

where $\Phi_{\mathrm{fr}}(B)$ is the radical yield in a magnetic field of flux density $B$.

\section{Discussion}

The general agreement between theoretical and experimental results as displayed in Figs. 6 and 7 must be regarded as strong evidence that the mechanism discussed here is operating in these systems. As can be seen, however, in Fig. 6 there seems to be a systematic discrepancy at intermediate field strength (about $1 \mathrm{k}$ Gauss) which is probably due to the simplified treatment of Eq. (10) viz. neglecting $H_{\mathrm{D}}$ and applying a rate constant treat-

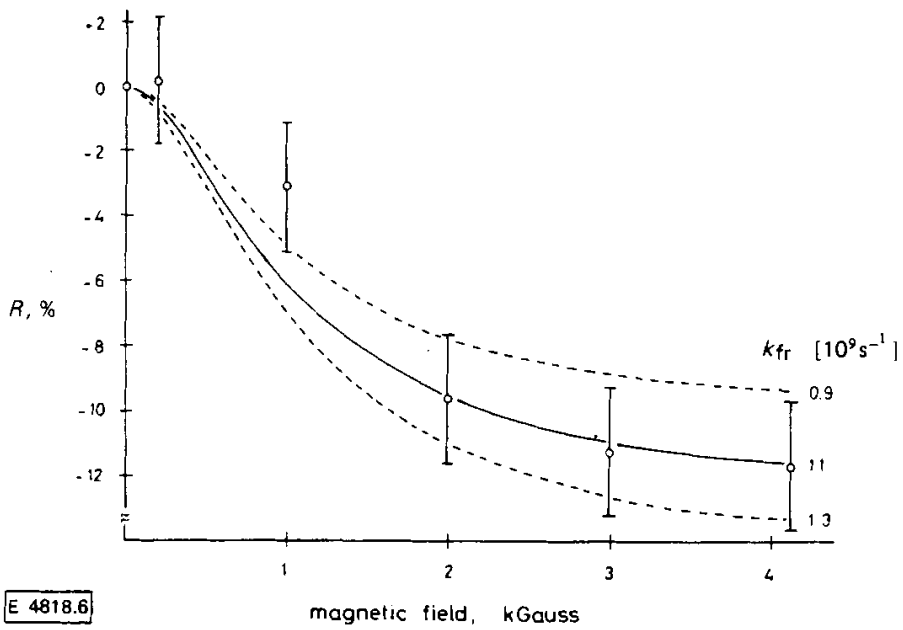

Fig. 6

Relative magnetic field effect $R$ (cf. Eq. (38)) as a function of magnetic flux density for the triplet exciplex thionine/p-iodoaniline. Circles with error bars represent experimental results from ref. [13], lines are calculated using Eqs. (35)-(38) and the different values for $k_{\mathrm{fr}}$ indicated in the diagram

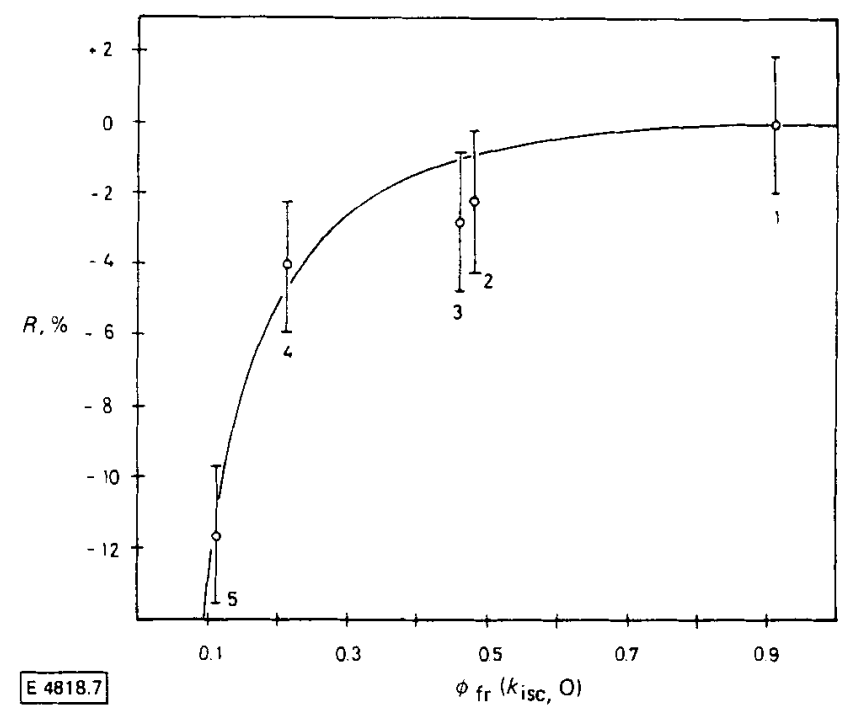

Fig. 7

Relative magnetic field effect $R$ (cf. Eq. (38)) at $4 \mathrm{k}$ Gauss as a function of radical yield at zero field $\Phi_{\mathrm{fr}}\left(k_{\mathrm{isc}}, 0\right)$. the numbers refer to different donors in the exciplex with thionine triplet. 1 aniline, $2 \mathrm{p}-\mathrm{Br}$-aniline, $3 \mathrm{~m}$-I-aniline, $4 \mathrm{o}$-I-aniline, $5 \mathrm{p}$-I-aniline. The solid line is calculated using Eqs. (35) $-(38)$ and $k_{\mathrm{fr}}=1.1 \cdot 10^{9} \mathrm{~s}^{-1}$

ment for the magnetic field influence. Especially the approximation applied in Eq. (34) must be considered as a first approach to the exact solution. It is clear from Fig. 5 that this approximation should tend to overestimate the magnetic field effect due to the discrepancy at short times. As can be seen from Fig. 6 the theoretical magnetic field effect at $1 \mathrm{k}$ Gauss is indeed stronger than the observed one. The limitation of the presently applied approximations will be discussed in detail on the basis of an exact numerical solution of Eq. (10) [28].

It will be important to note that the mechanism described in this paper is in principle not restricted to the case of triplet exciplexes. It may be assumed to operate in any case where a chemically reactive triplet state is subject to sublevel selective 
intersystem crossing, which may be enhanced by suitable heavy atom substitution. Possible photochemical reactions which may in this way become sensitive to magnetic field influence could be hydrogen atom transfer or proton transfer and also cis-trans isomerisations. the exploitation of such effects can provide information on the sublevel dynamics and absolute rate constants of short lived triplet intermediates in the nanosecond and subnanosecond region not accessible to phosphorescence and esr-techniques.

In 1972 Gupta and Hammond [29] reported a magnetic field effect on carbonyl triplet sensitized cis-trans isomerisations for which they could not give a consistent interpretation but suspected that it might be due to a magnetic field effect on the internal conversion process (intersystem crossing) of an intermediate exciplex or on the relaxation among the three sublevels of the exciplex triplet. Atkins [30] tried to explain these effects in terms of a $\Delta g$-mechanism as occurring similarly in radical pairs, but a quantitative test of this mechanism was not applied since the experimental results did not give information on the explicit magnetic field strength dependence of the effects. It would be of interest to reinvestigate the effects described by Gupta and Hammond [29] in the light of the present mechanism.

Finally we should like to comment on the relation between the triplet mechanism of CIDEP and the mechanism discussed in this paper. In both cases the starting point is a triplet spinpolarization in the molecular frame of a molecule oriented randomly and diffusing in solution. Whereas in the CIDEP triplet mechanism this spin-polarization in the molecular frame is brought about by a spin-selective population process, in our case there is selective depopulation of the triplet sublevels.

Secondly the role of the zero field splitting of the triplet sublevels is essentially different in both mechanisms. Whereas in the CIDEP triplet mechanism non-vanishing zero-field splitting is a necessary condition that spin-polarisation is transferred from the molecular frame to the laboratory frame $[31,32]$ to be ultimately detectable as electron spin-polarisation in the esr-spectrum of radicals generated from the triplet, the zero-field splitting parameter in our case is of secondary importance. This may be noted from the fact that in our approximative treatment of the mechanism it could be completely neglected. It may be expected, however, that a non-vanishing zero-field splitting, in our case too, will lead to a detectable electron spin-polarisation of the radicals formed in the dissociation of the exciplex.

\section{References}

[1] P. W. Atkins and T. P. Lambert, Ann. Rep. Prog. Chem. A 72, 67 (1975).

[2] A. L. Buchachenko, Russ. Chem. Rev. (Uspekhi Khim.) 45, 761 (1976).

[3] Y. N. Molin, R. Z. Sagdeev, and K. M. Salikhov, Sov. Sc. Rev. B: Chem. Rev. I, 1 (1979), Ed. M. E. Voplin.

[4] A. Lepley and G. L. Closs, Chemically Induced Magnetic Polarization, Wiley, New York 1973.

[5] L. T. Muus, P. W. Atkins, K. A. McLauchlan, and J. B. Pedersen, Chemically Induced Magnetic Polarization, Proc. NATO Adv. Study Inst., Sogesto Urbino, Italy, Reidel, Dordrecht, Holland, 1977.

[6] F. J. Adrian, Rev. Chem. Intermed. 3, 3 (1979).

[7] J. K. S. Wan, Adv. Photochem. 12, 283 (1980), Eds. J. N. Pitts, Jr., G. S. Hammond, and K. Gollnick.

[8] S. K. Wong, D. A. Hutchinson, and J. K. S Wan, J. Chem. Phys. 58,985 (1973)

[9] P. W. Atkins and G. T. Evans, Mol. Phys. 27, 1633 (1974).

[10] F. J. Adrian, J. Chem. Phys. 61, 4875 (1974).

[11] J. B. Pedersen and J. H. Freed, J. Chem. Phys. 62, 1706 (1975).

[12] U. Steiner, Chem. Phys. Lett. 74, 108 (1980).

[13] U. Steiner, Z. Naturforsch. 34a, 1093 (1979).

[14] U. Steiner and G. Winter, Chem. Phys. Lett. 5.5, 364 (1978).

[15] K. F. Freed, Radiationless Processes, Topics in Applied Physics, Vol. 15, p. 23, Ed. F. K. Fong, Springer, Berlin 1976.

[16] U. Steiner, Schweratome als moleculare Sonden zum Nachweis und Studium des Verhaltens von Triplettexciplexen, Hochschulverlag, Freiburg 1979.

[17] J. Friedrich, F. Metz, and F. Dörr, Mol. Phys. 30, 289 (1975).

[18] J. Friedrich, G. Weinzierl, and F. Dörr, Z. Naturforsch. 3la, 748 (1976).

[19] J. H. Freed, G. V. Bruno, and C. F. Polnaszek, J. Phys. Chem. 75,3385 (1971).

[20] R. Kubo, J. Phys. Soc. Jpn. Suppl. 26, 1 (1969).

[21] J. Freed, G. V. Bruno, and C. F. Polnaszek, J. Chem. Phys. 55, 5270 (1971).

[22] H. Beens, J. deJong, and A. Weller, Colloque Ampère 15, Ed. P. Averbuch, North-Holland, Amsterdam 1969.

[23] H. Hayashi, S. Iwata, and S. Nagakura, J. Chem. Phys. 50, 993 (1969).

[24] C. P. Keijzers and D. Haarer, J. Chem. Phys, 67, 925 (1977).

[25] P. Debye, Polar Molecules, Dover Publications, New York 1945.

[26] U. Fano and G. Racah, Irreducible Tensorial Sets, Academic Press, New York 1959.

[27] In Eq. (17) we use the rotation matrices as defined in A. Messiah, Quantum Mechanics, Vol. 2, North-Holland, Amsterdam 1962.

[28] U. Steiner (to be published).

[29] A. Gupta and G. S. Hammond, J. Chem. Phys. 57, 1789 (1972).

[30] P. W. Atkins, Chem. Phys. Lett. 18, 355 (1973).

[31] P. W. Atkins, in Ref. [5], p. 191.

[32] P. J. Hore, Chem. Phys. Lett. 69, 563 (1980). 